\title{
Efficacy of the Manchester Short Splint versus traditional dorsal splint for rehabilitation of Zone II flexor tendon repairs: study protocol for a randomized controlled trial
}

Vivien Lees ( $\square$ vivienlees@protonmail.com )

Manchester University NHS Foundation Trust https://orcid.org/0000-0001-9928-6157

Denise O'Brien

MUFHT

Karen Redvers-Chubb

MUFHT

Study protocol

Keywords: Short Splint, zone II, flexor tendon repair, rehabilitation, regimen

Posted Date: September 4th, 2020

DOI: https://doi.org/10.21203/rs.3.rs-21059/v1

License: (c) (i) This work is licensed under a Creative Commons Attribution 4.0 International License.

Read Full License 


\section{Abstract}

Background: A previously reported audit of use of the Manchester Short Splint versus the traditional dorsal forearm-based splint for rehabilitation of zone II flexor tendon repairs suggested that patient outcomes were enhanced by use of Manchester Short Splint. Specifically, there was less flexion contracture at the proximal interphalangeal joints at 6 and 12 weeks with greater final range of motion (Strickland assessment). Importantly, there was no evidence of excess tendon ruptures complicating use of the new splint.

Methods: The present study is designed as a single-centre randomized controlled trial to compare the two splints [Clinical Evidence Level 2 as patient and treating therapists are aware of which splint is in use. Surgery is standardized with respect to the type of repair used. Inclusion criteria for the trial include single finger flexor tendon injury of zone II with up to one but not two simultaneous digital nerve injuries operated on within 4 days of the injury date. Exclusions include children, more complex injuries, those unable to give consent or otherwise co-operate with the trial protocol. Patients are recruited at their first hand therapy visit having been provided with information about the trial by one of the Site Investigators during their hospital admission. The consenting patient is randomized into one of two splint groups. Assessments recorded in the Case Report Form include range of joint motion (Strickland), PROMs (Quick Dash), visual analogue pain scales, tendon rupture event and need for further surgical intervention. Data is captured weekly for first 6 weeks and again at 12 weeks. At 12 weeks grip strength and fatigue of grip strength are also measured.

Discussion: The present study aims to determine the efficacy of the short splint versus the traditional splint in rehabilitation of Zone II flexor tendon repair. Our earlier published audit on the potential advantages of the short splint generated considerable interest internationally with numbers of hand therapy departments adopting the short splint in the absence of more substantive evidence. Clearly it is desirable that such evidence is forthcoming and the present study seeks to provide such evidence and inform development of a multi-centre study.

\section{Background}

The project sets out to compare two different splints in the outcome of zone II flexor tendon injury hand therapy rehabilitation program. Traditional hand therapy is based on using a long dorsally forearm-based splint for 6 weeks in the zone II flexor tendon injuries (1-4). A novel shorter splint has been devised as an alternative to the traditional longer splint and we have previously reported on the same providing preliminary observational data supporting the safety and efficacy of the short splint in clinical use (5). There are cogent reasons for believing that permitting more wrist movement during rehabilitation will improve the range of finger movement through passive extension of the interphalangeal joints (tenodesis effects)(6,7). 
The traditional splint is a forearm-based dorsal thermoplastic splint that immobilizes the wrist in neutral position with a block to $30^{\circ}$ of metacarpophalangeal (MCP) joint extension. In contrast, the Manchester Short Splint extends from the proximal wrist crease to the finger tips and is placed on the dorsal aspect of the hand. The Manchester Short Splint permits maximal wrist flexion and up to $45^{\circ}$ of wrist extension with a block to $30^{\circ}$ of MCP joint extension.

It is appreciated that wearing a splint for a 6 week period is restrictive for patients who are unable to perform activities of daily living using the affected hand for that period. As surgical repair design has innovated and repairs are now stronger using multi-strand rather than 2 strand designs (8) it is important that rehabilitation protocols are adapted to take best advantage of stronger repairs to deliver better outcomes by the end of the treatment period (9).

The objective of the study is to determine which of the two splint designs should be preferred in the rehabilitation of zone II flexor tendon injury repairs.

\section{Methods/design}

\section{Aim, design and setting}

The study design is that of a single-blind prospective randomized comparison of two splinting methods for patients who have undergone zone II flexor tendon repair. The treating hand therapist and the patient would be aware of the treatment regimen but the assessing hand therapist (brought in for the 6 week and 12 week assessments) is specifically not aware of the splint used and remains 'blinded' to that information. The study is a single-center study that will inform development of a future multicenter study and is clinically based within the hand trauma service that serves a major conurbation and sees just under 4000 cases annually.

\section{Characteristics of participants-recruitment to study}

All patients who have sustained zone II flexor tendon injury and also meet the inclusion criteria of the study will be assessed by a Site Investigator (surgeon) for their potential suitability for the study and if appropriate will be invited to participate.

The detailed inclusion and exclusion criteria are presented in Table 1. These criteria include both patient factors and injury factors.

\section{Processes, interventions and comparisons}

The specific intervention is to test the use of a short splint against the traditional long forearm-based splint that we normally use for zone II flexor tendon repairs. Figure 1 illustrates the two splint designs with the traditional long dorsal splint (Fig.1A Dorsal view and Fig 1B lateral view) and the Short Splint (Fig. 1C 
Dorsal view and Fig 1D lateral view). The timelines, and assessments are detailed in SPIRIT Figure 2. This includes some modifications to the traditional exercise regime specifically to engage wrist movement and take advantage of the tenodesis phenomenon that passively increases interphalangeal joint extension. Detailed measurements are taken at 6 and 12 weeks into treatment in all patients by a 'blinded' therapist who is not party to which splint has been used (in all patients the splint is discontinued at 6 weeks). The frequency of visits to the hand therapy department is the same for both groups so there are no extra attendances as a result of participation in the trial.

The patients are counselled and invited to participate in the trial by a Site Investigator who is a member of the surgical team. The patient is provided with Patient Information Leaflet (Appendix 1). Recruitment only takes place at the first attendance to the hand therapy department when a therapist who is a Site Investigator goes through the counselling and consent for the trial and answers questions the patient may have. A Patient Consent Form is also completed at this time.

The surgery that is undertaken is by the standard protocol of the department and is not affected by this study. This protocol is for a 4-strand core suture using $3 / 0$ prolene and a $5 / 0$ epitendinous suture with adequate venting of the pulleys of the flexor tendon sheath. We are only concerned in this study with the aftercare.

It is normal practice to see the flexor tendon patients 4-5 days after surgery to commence rehabilitation. The patients in the study will have had this period of time to consider whether they wish to participate or not. If they choose to join the study then they are randomized at the first hand therapy visit by a hand therapist who is also one of the site investigators. The allocation model is of computer generated randomization sequence prepared at the start of the trial allocating patients into either Group A (Group $A$ controls traditional splint) or Group B (Group B Manchester short splint). Patients who choose not to enter the study receive the traditional splint. Patients in both groups are treated by an experienced team of specialised hand therapists who will supervise their rehabilitation.

A patient who has initially given consent to participate but then loses capacity or chooses to withdraw consent to participate is withdrawn from the study. In practice this means that a patient in Group B (Manchester Short Splint) converts to traditional splinting any time within the first 6 weeks after operation. A patient on Group A (traditional splint) continues with that splint but does not undergo the specific assessments of the study.

Follow up arrangements are the same for each group and all patients are required to attend therapy clinic once each week for the first 6 weeks and undertake specified exercises. Following removal of the splint at 6 weeks, a program of exercise, soft tissue stretching and night splinting for residual contracture is initiated. Patients in both groups are permitted to return to normal activities at 12 weeks. From 6 weeks onwards once the splint is removed the treatment of all patients, regardless of Group is the same.

The rehabilitation protocol for both groups is that of early combined passive flexion and active motion exercises. The exercise sequence emphasises full passive flexion stretches to maximise passive digital 
motion prior to commencing active motion. Active flexion exercises is initiated from the distal interphalangeal (DIP) joint to maximise differential glide. Patients are encouraged to perform active flexion exercises carefully to minimise the work of flexion. They are encouraged to perform active digital extension exercises to minimise the occurrence of interphalangeal joint flexion contractures. Furthermore, patients are discouraged from performing excessive or forced active flexion at the end range of motion.

Patients in group A (traditional splint) perform digital flexion and extension exercises within the forearmbased splint keeping the wrist in a neutral and MCP joints at $30^{\circ}$ flexion position. In contrast Patients in group B (Manchester short splint) perform active digital flexion exercises with the wrist extended to $45^{\circ}$ and active digital extension exercises with the wrist in maximal flexion with the MCP joints at $30^{\circ}$ flexion.

In both groups, volar thermoplastic finger splints will be provided for use at night in those patients who are unable to achieve full interphalangeal joint extension and therefore have incipient fixed flexion tendency notably of the PIPJs.

All patients will be given instructions on the safe and light functional use of their hands, excluding the injured digit(s). The patients will be instructed to wear their splints for a period of 6 weeks.

Assessment is undertaken at 6 weeks and on completion of treatment at 12 weeks by the 'blinded' hand therapist. The primary outcomes are the integrity of the tendon (presence or absence of tendon rupture) and range of motion of PIPJ and DIPJ of the affected finger and grip strength at 12 weeks only (grip strength at 6 week would risk tendon rupture). Secondary outcomes comprise Visual Analogue Scale for pain score, Quick DASH patient questionnaire, and the PEM questionnaire. The number and duration of visits to the hand therapy department is the same for both groups. The study duration is from the time the patient is first seen in the Hand Therapy clinic post-surgery and completes at 12 weeks for the patient.

Patients undergoing flexor tendon repair may potentially experience poor outcomes in terms of flexor tendon adhesions requiring flexor tenolysis or flexor tendon rupture requiring re-repair. In addition surgical wound infection can occur requiring surgical intervention to washout the flexor sheath. Need for such surgical interventions over and above the trial protocol will be recorded as adverse events within the Case Report Form and then the necessary surgery will take place as indicated. Additional surgeries may fall within the trial timeline, for example flexor tendon re-repair or after the monitoring period has concluded, for example flexor tenolysis. Patients affected in this way will still continue on the trial and have their data collected.

Table 2 details the assessments, investigations and interventions undertaken by the hand therapists with comparison on which parts of the pathway represent additional items that would not otherwise be part of routine treatment.

The assessment by the Site Investigator hand therapist includes:

- Quick DASH, PEM 
- pain scales (Numerical Analogue Scale 0-10)

- range of motion of injured digit using digital goniometer

- grip strength and endurance power grip at 12 weeks using Jamar dynamometer on 2nd setting (power grip is not tested earlier to avoid potential tendon rupture)

\section{Trial management}

The trials team will meet regularly to review conduct and progress of the trial and to ensure trial documentation is maintained in compliance with GCP requirements. The trials team will initiate and receive confirmation from the REC for trial amendments. Communication of approved changes will be communicated through the site investigators team by the Principal Investigator. This team will be scientifically independent from the sponsor. The responsibility for interim analyses and stopping guidelines will be with the trials team and Principal Investigator with advice from the trial statisticians.

Recruitment strategies will include regular educational updates for new members of the surgical team to act as Site Investigators. Our research nurse will assist with identification of potential participants based around the morning trauma meeting. The departmental trauma co-ordinator and trauma clinic nurses will also assist in case identification. In addition regular review of reasons for non-recruitment of potential patients will be undertaken. Retention of trial patients once on study is recognized to be important and this will be supported through the continuity of high level care in the hand therapy department.

Trial ethical approval has been obtained form the Research Ethics Committee 14/NW/1486 IRAS project ID 159054. The Research and Ethics Committee will send an independent reviewer to undertake a site visit to monitor trial conduct, compliance and documentation (approximately annually).

The Trust as sponsor is acting for ancillary and post-trial care as the need arises.

\section{Statistical analysis and power calculation}

Advice on statistical analysis was obtained from lead statistician Manchester University Foundation Hospitals Trust. On the basis of the observation from Peck et al 2014 (5) that there is a significantly reduced extension deficit at the PIP joint (median difference $=13$ and mean difference $=10$ ) and an increase in the total arc of flexion (median difference $=18$ and mean difference $=12$ ) for patients with the short splint compared to those with a traditional splint, then the advice is to recruit 60 patients (approx. 30 in each group). The outcomes in the original study (5) appear to follow close to a normal distribution such that a simple two-sample t-test should be used. The data set from the original study demonstrated SD of 21 and if it is assumed that that will be similar in the current study then the simple t-test will give a predicted power of $80 \%$ to detect the anticipated differences in anticipated improvements in pain and grip strength at the conventional $5 \%$ significance level. 


\section{Data management}

The study protocol will comply with standard Caldicott and GDPR regulations. No names or dates of birth are used with respect to data entry to the study. Special patient identifier numbers will be generated for the Case Record Form (CRF) and used to identify patients through the study. Data collection is manual and this raw data is then transcribed into Excel held on Trust computer. The CRF hard copy files will be maintained on site consistent with regulations. The raw data transcription into electronic format will be undertaken by member of the hand therapy team. Primary data will be processed on Trust computers by the authors and secondary data transported without patient identifiers on encrypted pen drives.

Processed data will be stored on home computers of the authors of the research study and will be the subject of peer review presentation and publication so will be communicated electronically at this stage.

Post study personal data will be stored for less than 12 months from the conclusion of the study (electronic and hard copy). Processed data will be stored in the Hand Therapy department files. Lead Hand Therapist (DB) will have access to the same and the processed data (electronic and hard copy) will be destroyed after 10 years. There are no specific plans to facilitate public access to the final trial dataset but this will be available on reasonable request from the Principal Investigator (VL).

Finally, publication of the results will be sought in peer-reviewed journal with presentation at national meetings. Summary of results will be communicated to participants by letter. The results will also be reviewed with our PI (patient involvement) group.

\section{Discussion}

Should the short splint be found to work better than the traditional long splint, then this will have important implications for how flexor tendon injuries are rehabilitated in future. Specifically, we are not looking at the safety and efficacy of the short splint with respect to 2-strand tendon repair techniques and confine our attention to 4-strand tendon repair techniques in the current study.

To date the principal challenges on the study have been those around recruitment. By definition the subjects of the study have sustained injury which requires treatment through the urgent care pathway of the trauma service and the wider department have needed to be involved in the identification of potential patients to the study. This has necessitated ongoing efforts to train staff members as site investigators expert in the conduct of the study.

The 'burdens' for the patient are minimal in this study with the same number of visits as would normally be offered for full rehabilitation of the flexor tendon injury. Individual sessions will take slightly longer to accommodate specific assessments and recording of those assessments.

Recruitment issues that have been encountered to date in the current study include considerable number of patients being excluded because of strict injury-related criteria for study entry. For example two fingers with flexor tendon injury may not be included in this study but there is no specific evidence that these 
have different outcomes from single finger injuries as long as the injury is otherwise not complex. It may be that in a future multicenter study there could be some adjustments to these injury-related criteria that would enhance recruitment. The patient-specific inclusion criteria are deemed to have been appropriate to date. A further issue that has arisen is the time restriction between injury and definitive surgery for the flexor tendon injury which is limited to 4 days in this study. This timeline is believed to be appropriate to avoid the confounding influence of wound infection supervening and leading to higher complication rates that would flag as adverse events on the study. It has not always been possible to manage patients on this timeline given pressures on the regional hand trauma service. As a consequence numbers of potential patients have had to be excluded for having exceeded the 4 day timeline.

The study design is that of single blind i.e. the treating surgeon and physiotherapist are likely to have a view on which regimen is working better before we come to the end of the study. It will be important to avoid conveying our own subjective impressions to patients who are on the study.

Retention of patients once on the study is also one of our challenges. The study design specifies assessments at 6 weeks and 12 weeks. The later time point was included after some discussion knowing that there would be some loss of patients to follow up. The reason to have included it is so that we could assess any early differences in range of movement / need for tenolysis procedure and assists our understanding how quickly patients were getting back to work and was therefore important for both clinical and socioeconomic reasons. It is believed that this time point needs to be retained in any future similar studies. This study has not been able to provide inconvenience payments to patients and would be something to consider in any suture study. With many patients having taken 8 weeks off work because of the initial injury the 12 week timepoint represents the period when they are likely to be back at work and under some pressure not to take more time off for hospital appointments.

If we can determine a better outcome with the shorter splint than the one we are using currently we may be able to significantly impact and improve patient outcomes not only in this country but also globally. The materials needed to make the short splint are widely available and the shorter splint can be fashioned using whatever local materials are available. The caveat would be that the surgical repair of the flexor tendon itself is by the multistrand repair which represents the current standard of care for Zone II flexor tendon repairs.

\section{Declarations}

\section{Trial Status}

Recruitment of first patient 31.3.15. Recruitment completed 6.4.20 subsequent to first submission of manuscript 3.4.20. Study is ongoing for subsequent patient review and measurements as protocol for the subsequent 12 week period and will conclude 29.6.20.

\section{Ethics approval and consent to participate}


North West Haydock Research Ethics Committee IRAS project ID: 159054

Trial registration: ClinicalTrials.gov Identifier: NCT03850210

21.2.19 Retrospectively registered

https://clinicaltrials.gov/ct2/results? cond $=$ \&term $=$ NCT03850210\&cntry $=$ \&state $=$ \&city $=$ \&dist $=$ See website for WHO Trial Registration Data Set

All patient participants undergo full informed consent process as part of the recruitment to the trial (Appendix 2-consent form).

\section{Consent for publication}

\section{Image Consent Publication Statement}

The images Figures 1A-D inclusive are of my own hand and I give consent for publication in the Trials Journal

Vivien Lees

1.5 .20

\section{Data availability statement}

The datasets generated and/or analysed during the current study are not publicly available due but are available from the corresponding author on reasonable request.

\section{Competing interests}

none to declare

\section{Funding}

No grant was sought or awarded for this study. The study has been registered. The Trust will cover the costs of Investigators time as they undertake their trial roles alongside their regular duties. There will be no payments to participants, investigators or Lead Investigator.

\section{Authors contributions}

"The authors declare that they have no competing interests" 


\section{Acknowledgements}

Authors listed on this publication have made contribution in line with the expected contributions specification.

In addition the authors acknowledge Fiona Peck for work in development of the short splint design, Emma Birkin and Alison Roe for their contributions in the earlier parts of the study. All Site Investigators and colleagues for assisting in recruitment of patients to the study. Bradley Tallon for trial support. Department of Medical Statistics for power calculation.

\section{References}

1. Peck FH, Bucher CA, Watson JS, Roe A. A comparative study of two methods of controlled mobilization of flexor tendon repairs in zone 2. J Hand Surg Br. 1998, 23: 41-5.

2. Thien TB, Becker JH, Theis JC. Rehabilitation after surgery for flexor tendon injuries in the hand. Cochrane Database Syst Rev. 2004: CD003979.

3. Small JO, Brennen MD, Colville J. Early active mobilisation following flexor tendon repair in zone 2. J Hand Surg Br. 1989, 14: 383-91

4. Neiduski RL, Powell RK. Flexor tendon rehabilitation in the $21^{\text {st }}$ century: A systematic review. J Hand Therapy 2019; 32: 165-174.

5. Peck F, Roe AE, Ng CY, Duff C, McGrouther DA, Lees VC. The Manchester short splint: A change to splinting practice in the rehabilitation of zone II flexor tendon repairs. Hand Therapy 2014; 19(2) : 4753.

6. Savage R. The influence of wrist position on the minimum force required for active movement of the interphalangeal joints. J Hand Surg Br 1988; 13(3): 262-8.

7. Woythal L, Homer P, Brorson S. Splints, with or without wrist immobilization, following surgical repair of flexor tendon lesions of the hand: A systematic review. Hand Surg and Rehab 2019; 38: 217-222

8. Kaskutas V, Powell R. The impact of flexor tendon rehabilitation restrictions on individuals' independence with daily activities: implications for hand therapists. J Hand Ther. 2013, 26: 22-8; quiz 9

9. Hardwick JT, Tan JJ, Foster MA and Titley OG. A systematic review of 2-strand versus multistrand core suture techniques and functional outcome after digital flexor tendon repair. J Hand Surg Am 2014; 39(4): 686-695.

\section{Tables}

Table 1 Inclusion and exclusion criteria 


\begin{tabular}{|ll|}
\hline INCLUSION CRITERIA & EXCLUSION CRITERIA \\
\hline $\begin{array}{l}\text { Adult patient (16 yrs or older) } \\
\text { without FDS division. Includes single nerve injury } \\
\text { on same digit. }\end{array}$ & $\begin{array}{l}\text { Patients with special needs and vulnerable } \\
\text { groups }\end{array}$ \\
\hline $\begin{array}{l}\text { Time interval of injury to operation }<96 \text { hours (4) } \\
\text { days) }\end{array}$ & $\begin{array}{l}\text { Patients lacking capacity or motivation to } \\
\text { participate in planned physiotherapy }\end{array}$ \\
\hline $\begin{array}{l}\text { Patients unable to attend physiotherapy for } \\
\text { requisite number of sessions for social and } \\
\text { economic reasons }\end{array}$ \\
$\begin{array}{l}\text { Patients unable to understand English sufficient } \\
\text { to complete rehabilitation }\end{array}$ \\
$\begin{array}{l}\text { Flexor tendon injury greater than } 4 \text { days previous } \\
\text { Two digital nerve injuries in the same finger as } \\
\text { the flexor tendon injury }\end{array}$ \\
$\begin{array}{l}\text { More than one finger with FDP injury } \\
\text { Multilevel FDP injury in one finger }\end{array}$ \\
\hline Complex injury with soft tissue defect \\
\hline Complex injury with underlying fracture \\
\hline
\end{tabular}

Table 2 Details of assessments and interventions undertaken by the hand therapists 
First postoperative visit (within 5 days of flexor tendon repair operation)

Fashioning of thermoplastic splint. Advice to patient on exercises to be undertaken. Safe use of the hand.

*Recruitment into trial with counselling regarding same. Trial consent form is completed and patient is randomized to short splint (intervention) or long traditional splint (control)

Patient reviewed weekly thereafter at 2,3,4, and 5 weeks. During visit the patient is further advised with supervised exercises. Integrity and glide of tendon repair are assessed. Same for trial and nontrial patients.

*Trial patients - any adverse events are reported in CRF

6 week review. Splint is discontinued and the range of motion of the affected finger is made and recorded using goniometer. Integrity and glide of tendon repair are assessed. Advice is given on driving and return to normal activities of daily living and sporting and recreational activities. Data is recorded.

*Trial patients have similar input but their measurements are performed by a blinded hand therapist after removal of the splint (single blind protocol). Any adverse events are reported in CRF. In addition the PEM, Quick DASH and VAS PROMs scales are administered and recorded in the CRF.

12 week review. Patients who attend will have their range of motion and grip strength recorded using Jamar dynamometer on the second setting. Integrity and glide of tendon repair are assessed. Data is recorded.

*Trial patients have similar input but their measurements are performed by a blinded hand therapist after removal of the splint (single blind protocol). Any adverse events are reported in CRF In addition the PEM, Quick DASH and VAS PROMs scales are administered and recorded in the CRF.

*Comments in italics highlight those parts of the pathway representing additional items that would not otherwise be part of routine treatment

\section{Figures}




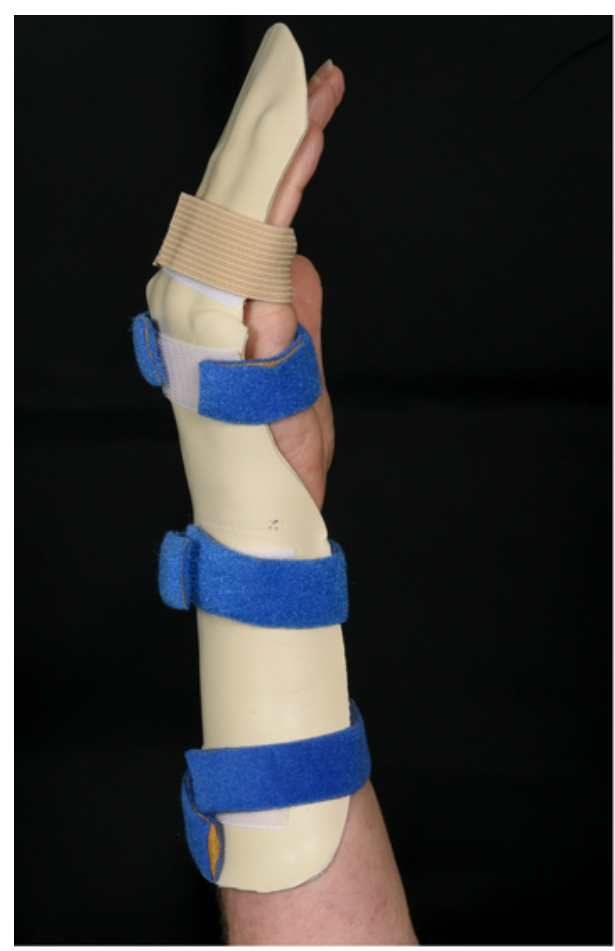

A

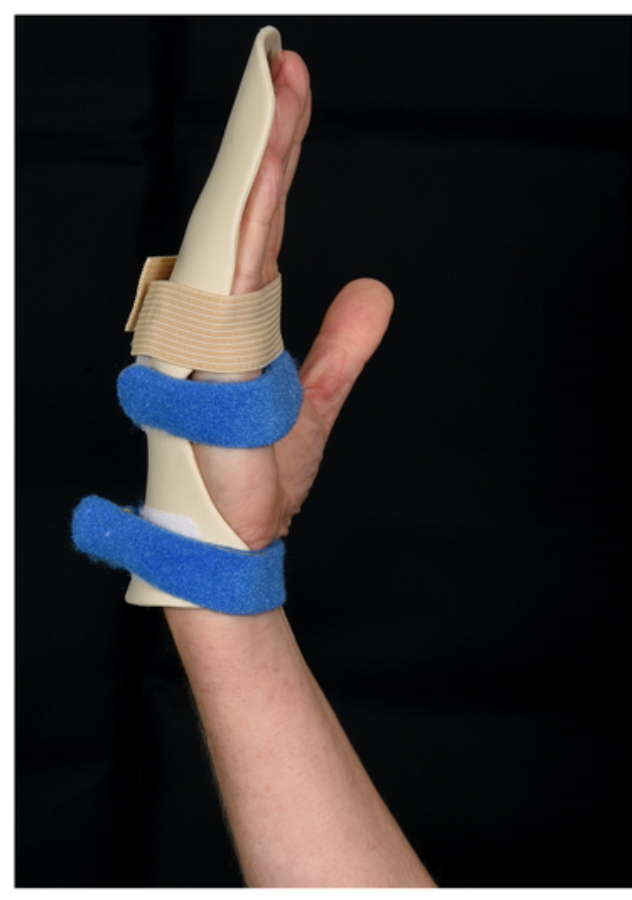

C

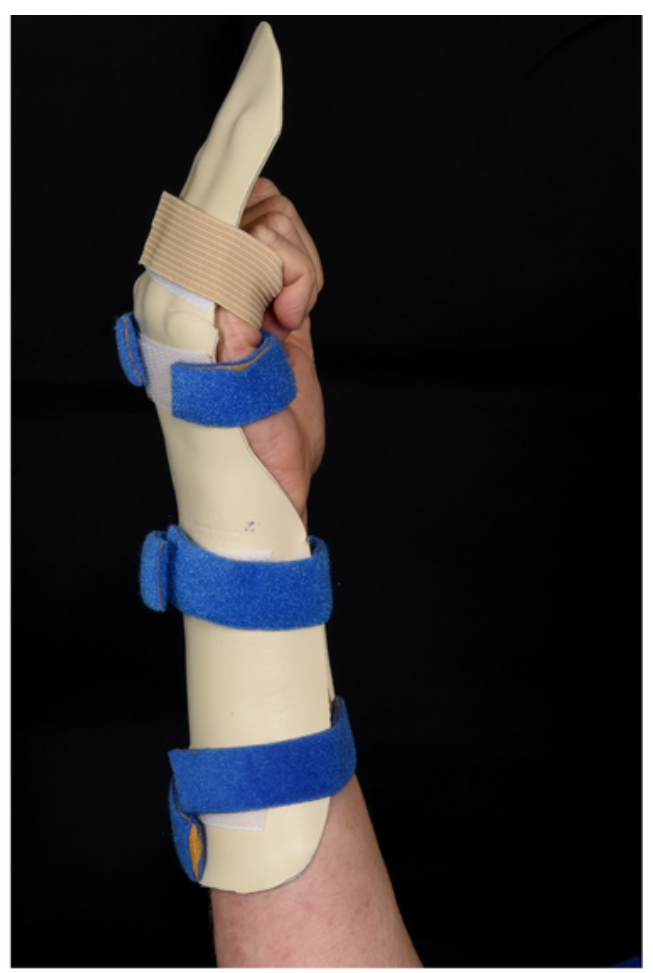

B

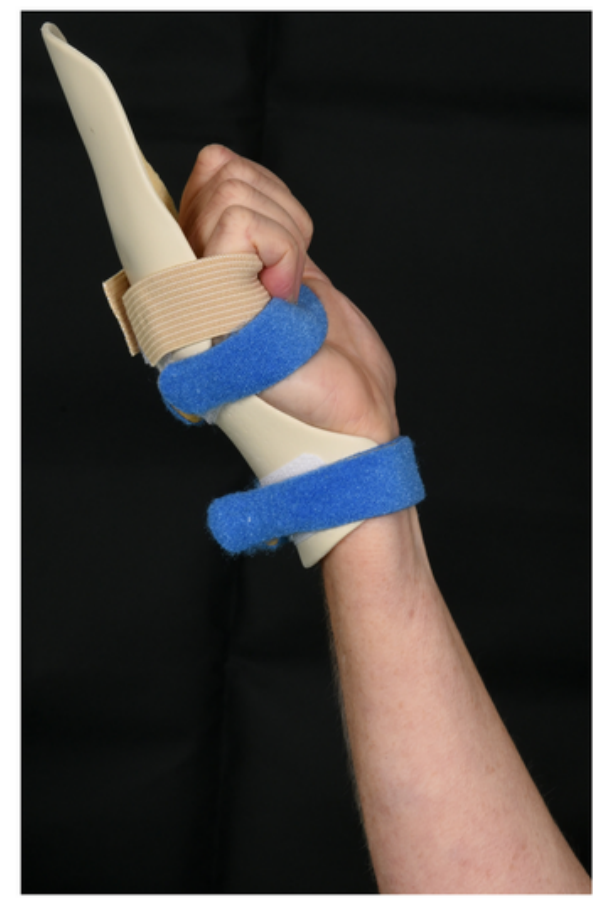

D

\section{Figure 1}

The two splint designs 


\begin{tabular}{|c|c|c|c|c|c|}
\hline & \multicolumn{5}{|c|}{ STUDY PERIOD } \\
\hline \multirow[b]{2}{*}{ TIMEPOINT"* } & Enrolment & Allocation & \multicolumn{2}{|c|}{$\begin{array}{l}\text { Post-allocation } \\
\text { a8sesemente }\end{array}$} & \multirow{2}{*}{$\begin{array}{c}\text { Clooe-out } \\
t_{t} \\
12 \text { weeks }\end{array}$} \\
\hline & $\begin{array}{c}\leq_{4 \text { days }} \\
\text { surgery to hand } \\
\text { theropy }\end{array}$ & 0 & $\begin{array}{c}t_{i} \\
6 \text { weeks }\end{array}$ & $\begin{array}{c}t_{2} \\
12 \text { weeks }\end{array}$ & \\
\hline ENROLMENT: & & & & & \\
\hline Ellgibilty screan & $\begin{array}{c}\mathrm{X} \\
\text { At presentation } \\
\text { for surgical } \\
\text { repgir of flexor } \\
\text { tendon }\end{array}$ & & & & \\
\hline $\begin{array}{l}\text { Paventr advised } \\
\text { abour walal and } \\
\text { provided with } \\
\text { Informadon laaner }\end{array}$ & $\begin{array}{c} \\
\text { Patient } \\
\text { approached } \\
\text { and given } \\
\text { information } \\
\text { about trial. } \\
\text { Cooling off } \\
\text { period prior to } \\
\text { Hand Therspy } \\
\text { visit }\end{array}$ & & & & \\
\hline Allocattion & & $\begin{array}{c}\text { X } \\
\text { At first } \\
\text { postoperative } \\
\text { visit to Hand } \\
\text { Therapy }\end{array}$ & & & \\
\hline Intormed coneent & & $\begin{array}{c}\text { X } \\
\text { Consent is taken } \\
\text { at time of } \\
\text { recruitment to } \\
\text { trisl at first Hand } \\
\text { Therapy visit }\end{array}$ & & & \\
\hline INTERVENTIONS: & & & & & \\
\hline $\begin{array}{l}\text { [Allocatiod to } \\
\text { rradtional vs short } \\
\text { spulling groups] }\end{array}$ & & $x$ & & & \\
\hline $\begin{array}{r}\text { ASSESSMENT S: } \\
\text { Range or movion } \\
\text { measure, Quick } \\
\text { Dash, PEIN, analog } \\
\text { pain scales] }\end{array}$ & & & $\mathrm{x}$ & $\mathrm{x}$ & $x$ \\
\hline $\begin{array}{r}\text { [Power grip } \\
\text { measurement with } \\
\text { Jamar } \\
\text { dynamometer] }\end{array}$ & & & & $x$ & $x$ \\
\hline
\end{tabular}

\section{Figure 2}

SPIRIT Figure. Schedule of enrolment, intervention and assessments.

\section{Supplementary Files}

This is a list of supplementary files associated with this preprint. Click to download. 
- AppendixA1ManchesterShortSplintInformationSheetV4iNov2019.doc

- AppendixA3ProtocolManchesterShortSplintV5.doc

- AppendixBV3SPIRITFillablechecklistShortSplint.doc

- QuickDASHEnglish.pdf 